\title{
MASS EQUIDISTRIBUTION FOR RANDOM POLYNOMIALS
}

\author{
TURGAY BAYRAKTAR
}

\begin{abstract}
The purpose of this note is to study asymptotic zero distribution of multivariate random polynomials as their degrees grow. For a smooth weight function with super logarithmic growth at infinity, we consider random linear combinations of associated orthogonal polynomials with subgaussian coefficients. This class of probability distributions contains a wide range of random variables including standard Gaussian and all bounded random variables. We prove that for almost every sequence of random polynomials their normalized zero currents become equidistributed with respect to a deterministic extremal current. The main ingredients of the proof are Bergman kernel asymptotics, mass equidistribution of random polynomials and concentration inequalities for subgaussian quadratic forms.
\end{abstract}

\section{INTRODUCTION}

Let $\varphi: \mathbb{C}^{m} \rightarrow \mathbb{R}$ be a $\mathscr{C}^{1,1}$ weight function (i.e. $\varphi$ is differentiable and all of its first partial derivatives are locally Lipschitz continuous) satisfying

$$
\varphi(z) \geq(1+\epsilon) \log \|z\| \text { for }\|z\| \gg 1
$$

for some fixed $\epsilon>0$. We define an inner product on the space $\mathcal{P}_{n}$ of multi-variable polynomials of degree at most $n$ by setting

$$
\langle p, q\rangle_{n}:=\int_{\mathbb{C}^{m}} p(z) \overline{q(z)} e^{-2 n \varphi(z)} d V_{m}(z)
$$

where $d V_{m}$ denotes the Lebesgue measure on $\mathbb{C}^{m}$. We also let $\left\{P_{j}^{n}\right\}_{j=1}^{d_{n}}$ be a fixed orthonormal basis (ONB) for $\mathcal{P}_{n}$ with respect to the inner product (1.2). A random polynomial is of the form

$$
f_{n}(z)=\sum_{j=1}^{d_{n}} c_{j}^{n} P_{j}^{n}(z)
$$

where $c_{j}^{n}$ are independent identically distributed (iid) real or complex subgaussian random variables (see $\S 3.3$ ) and $d_{n}:=\operatorname{dim}\left(\mathcal{P}_{n}\right)=\left(\begin{array}{c}n+m \\ n\end{array}\right)$. This allows us to endow $\mathcal{P}_{n}$ with a $d_{n}$-fold product probability measure $\operatorname{Prob}_{n}$ induced by the probability law of $c_{j}^{n}$. We also consider the product probability space $\prod_{n=1}^{\infty}\left(\mathcal{P}_{n}, \operatorname{Prob}_{n}\right)$ whose elements are sequences of random polynomials of increasing degree. We are interested in limiting distribution of zeros of random polynomials.

In the present setting, the choice of weight function $\varphi$ determines a weighted global extremal function $\varphi_{e}$ (see 2.2) which induces a weighted equilibrium measure $\mu_{e}$ (see 3.3) whose support is a compact set denoted by $S_{\varphi}$. The following result indicates that for a typical (in the sense of probability) sequence $\left\{f_{n}\right\}_{n=1}^{\infty}$ of random polynomials the masses (respectively, normalized zero currents) are asymptotic to the equilibrium measure (respectively, the extremal current):

Date: November 28, 2019.

2000 Mathematics Subject Classification. 32A60,32A25,60D05.

Key words and phrases. Random polynomial, equidistribution of zeros, equilibrium measure, global extremal function, Bergman kernel asymptotics.

T. Bayraktar is partially supported by TÜBİTAK grants BİDEB-2232/118C006, ARDEB-3501/118F049 and Science Academy BAGEP grant. 
Theorem 1.1. Let $\varphi: \mathbb{C}^{m} \rightarrow \mathbb{R}$ be a $\mathscr{C}^{1,1}$-weight function satisfying (1.1). Assume that random coefficients $c_{j}^{n}$ are iid real or complex subgaussian random variables of mean zero and unit variance. Then almost surely in $\prod_{n=1}^{\infty}\left(\mathcal{P}_{n}\right.$, Prob $\left._{n}\right)$ the masses

$$
\frac{1}{d_{n}}\left|f_{n}(z)\right|^{2} e^{-2 n \varphi(z)} d V_{m} \rightarrow d \mu_{\varphi_{e}}
$$

in the weak-star topology of measures on $S_{\varphi}$. Moreover, almost surely in $\prod_{n=1}^{\infty}\left(\mathcal{P}_{n}\right.$, Prob $\left._{n}\right)$ the normalized currents of integrations

in the sense of currents.

$$
\frac{1}{n}\left[Z_{f_{n}}\right] \rightarrow d d^{c} \varphi_{e}
$$

Distribution of zeros of random polynomials is a classical subject which goes back to Kac [Kac43] and Hammersley [Ham56] among others. A classical result due to Kac and Hammersley asserts that normalized zeros of Kac random polynomials (i.e. $\sum_{j=0}^{n} c_{j} z^{j}$ with iid Gaussian coefficients) of large degree tend to accumulate on the unit circle $S^{1}=\{|z|=1\}$. This ensemble of random polynomials has been extensively studied (see eg. [LO43, ET50, SV95, HN08, IZ13, Pri18] and references therein). In [SZ99], Shiffman and Zelditch obtained a far reaching generalization of the aforementioned result in the line bundle setting. Following [SZ99], asymptotic distribution of zero divisors of multi-variable random polynomials with random coefficients has been studied by various authors. In [Blo05, BS07] Bloom and Shiffman (see also [Bay17a]) considered random polynomials with Gaussian coefficients. Random polynomials with non-Gaussian coefficients were also considered by various authors (see eg. [DS06, BL15, Bay16, Bay17b, BD18] among others). In [Bay19] for radially symmetric weight functions, we provided a necessary and sufficient condition on random coefficients for equilibrium distribution of zero divisors of random polynomials (see also [BCM] for the line bundle setting). We refer the reader to the recent survey [BCHM18, $\S 2]$ for the state of the art.

Mass asymptotics have been considered by several authors in various geometric settings. Given a compact Kähler manifold $(M, \omega)$ of dimension $m$ and a positive Hermitian holomorphic line bundle $(L, h)$ whose curvature form $c_{1}(L, h)=\omega$, one can define a scalar $L^{2}$-product and a norm on the vector space of global holomorphic sections $H^{0}\left(X, L^{\otimes n}\right)$ by

$$
\|s\|_{n}^{2}:=\int_{M}|s(x)|_{h \otimes n}^{2} d V
$$

where $d V$ is the probability volume form induced by $\omega$. In [SZ99] Shiffman and Zelditch proved that for a sequence $s_{n} \in H^{0}\left(X, L^{\otimes n}\right)$ of global holomorphic sections of unit norm if their masses

$$
\left|s_{n}(x)\right|_{h \otimes n}^{2} d V \rightarrow d V
$$

in the weak-star topology of measures on $M$, the normalized zero currents $\frac{1}{n}\left[Z_{s_{n}}\right]$ (along zero divisors of $s_{n}$ ) converge weakly to the curvature form $\omega$ (see $\S 5$ for details). This was first observed by Nonnenmacher and Voros [NV98] in the case of the theta bundle over an elliptic curve $\mathbb{C} / \mathbb{Z}^{2}$. In a different direction, Rudnick [Rud05] proved a similar result in the setting of $S L_{2}(\mathbb{Z})$ modular cusp forms of weight $2 n$. The latter corresponds to the case of positive line bundle on a non-compact Riemann surface. All of the aforementioned results are based on potential theory.

In the $\mathbb{C}^{m}$ setting the result of [SZ99] corresponds to the case where the weight function is a Kähler potential (e.g. $\varphi(z)=\frac{1}{2} \log \left[1+\|z\|^{2}\right]$ ). The later implies that the weighted equilibrium measure is the restriction of a volume form defined on the complex projective space $\mathbb{P}^{m}$. More recently, Zelditch [Zel18] obtained a generalization to partially positive metrics on positive line bundles. However, the argument in [Zel18, Theorem 1.2] has a gap. Namely, it proves the $L_{l o c}^{1}$ convergence of the potentials only in the support of the Monge-Ampère measure. In order to 
complete the proof one needs to use a generalized domination principle (Theorem 2.2). We adapt the argument in [Zel18] to the current setting and fill this gap. Moreover, we generalize the mass equidistribution of random polynomials with subgaussian coefficients by using Hanson-Wright inequality [RV13] for subgaussian quadratic forms.

The outline of the paper is as follows: In $\S 2$ we prove that mass asymptotics implies equilibrium distribution of zeros (Theorem 2.4). In $\S 3$, we review near and off diagonal Bergman kernel asymptotics in the special case $Y=\mathbb{C}^{m}$ and a $\mathscr{C}^{1,1}$-weight function $\varphi: \mathbb{C}^{m} \rightarrow \mathbb{R}$ that has super logarithmic growth at infinity and apply these results to study associated Toeplitz operators and distribution of their eigenvalues. In $\S 3.4$, we prove Theorem 1.1. In $\S 4$ we discuss analogous results for random orthonormal bases. Finally, in $\S 5$ we give a generalization of Theorem 1.1 to the line bundle setting.

\section{Mass Asymptotics of Weighted Polynomials}

Let $Y \subset \mathbb{C}^{m}$ be a closed set and $\varphi: Y \rightarrow \mathbb{R}$ be a continuous weight function. If $Y$ is unbounded we assume that there exists $\epsilon>0$ such that

$$
\varphi(z) \geq(1+\epsilon) \log \|z\| \text { for }\|z\| \gg 1 .
$$

Following [ST97, Appendix B] we denote the weighted global extremal function

$$
V_{Y, \varphi}(z):=\sup \left\{u(z): u \in \mathcal{L}\left(\mathbb{C}^{m}\right), u \leq \varphi \text { on } Y\right\}
$$

where $\mathcal{L}\left(\mathbb{C}^{m}\right)$ denotes the Lelong class of pluri-subharmonic (psh) functions $u$ that satisfies

$$
u(z)-\log ^{+}\|z\|=O(1)
$$

where $\log ^{+}=\max (\log , 0)$. We remark that when $Y$ is compact and $\varphi \equiv 0$ (i.e. in the unweighted case) the extremal function defined in (2.2) is the pluri-complex Green function of $Y$ (cf. [Kli91]) and denoted by $V_{Y}$. We also denote by

$$
\mathcal{L}^{+}\left(\mathbb{C}^{m}\right):=\left\{u \in \mathcal{L}\left(\mathbb{C}^{m}\right): u(z) \geq \log ^{+}\|z\|+C_{u} \text { for some } C_{u} \in \mathbb{R}\right\} .
$$

In what follows, we let

$$
g^{*}(z):=\limsup _{w \rightarrow z} g(w)
$$

denote the upper semi-continuous regularization of $g$. Seminal results of Siciak and Zaharyuta (see [ST97, Appendix B] and references therein) assert that $V_{Y, \varphi}^{*} \in \mathcal{L}^{+}\left(\mathbb{C}^{m}\right)$ and that $V_{Y, \varphi}$ verifies

$$
V_{Y, \varphi}(z)=\sup \left\{\frac{1}{\operatorname{deg} p} \log |p(z)|: p \text { is a polynomial and } \sup _{z \in Y}|p(z)| e^{-\operatorname{deg}(p) \varphi(z)} \leq 1\right\} .
$$

For $r>0$ let us denote $Y_{r}:=\{z \in Y:\|z\| \leq r\}$. It is well-known that $V_{Y, \varphi}=V_{Y_{r}, \varphi}$ for sufficiently large $r$ ([ST97, Appendix B, Lemma 2.2]).

A closed set $Y \subset \mathbb{C}^{m}$ is said to be locally regular at $w \in Y$ if for every $\rho>0$ the extremal function $V_{Y \cap \overline{B(w, \rho)}}(z)$ is continuous at $w$. The set $Y$ is called locally regular if $Y$ is locally regular at every $w \in Y$. A classical result of Siciak [Sic81] asserts that if $Y$ is locally regular and $\varphi$ is continuous weight function then the weighted extremal function $V_{Y, \varphi}$ is also continuous and hence $V_{Y, \varphi}=V_{Y, \varphi}^{*}$ on $\mathbb{C}^{m}$. In the rest of this section we assume that $Y$ is a locally regular closed set.

The psh function $V_{Y, \varphi}$ is locally bounded on $\mathbb{C}^{m}$ and hence by Bedford-Taylor theory [BT76, BT82] the weighted equilibrium measure

$$
\mu_{Y, \varphi}:=\frac{1}{m !}\left(d d^{c} V_{Y, \varphi}\right)^{m}
$$


is well-defined and does not put any mass on pluripolar sets. Here; $d=\partial+\bar{\partial}$ and $d^{c}:=\frac{i}{2 \pi}(\bar{\partial}-\partial)$ so that $d d^{c}=\frac{i}{\pi} \partial \bar{\partial}$ and

$$
\int_{\mathbb{C}^{m}}\left(d d^{c} \frac{1}{2} \log \left[1+\|z\|^{2}\right]\right)^{m}=1
$$

Moreover, denoting the support $S_{Y, \varphi}:=\operatorname{supp}\left(\mu_{Y, \varphi}\right)$ by [ST97, Appendix B] we have

$$
S_{Y, \varphi} \subset\left\{z \in Y: V_{Y, \varphi}(z)=\varphi(z)\right\} \text {. }
$$

Thus, the support $S_{Y, \varphi}$ is a compact set. We denote its interior (as a subset of $\mathbb{C}^{m}$ ) by $\operatorname{Int}\left(S_{Y, \varphi}\right)$. An important example is $\varphi(z)=\frac{\|z\|^{2}}{2}$ which in turn gives $\mu_{Y, \varphi}=\mathbb{1}_{B} d V_{m}$ where $\mathbb{1}_{B}$ denotes the characteristic function of the unit ball in $\mathbb{C}^{m}$.

A locally finite measure $\nu$ is called a Bernstein-Markov (BM) measure for the weighted set $(Y, \varphi)$ if for sufficiently large $r>0$ the triple $\left(Y_{r}, Q, \nu\right)$ satisfies the weighted Bernstein-Markov inequality. That is, there is $M_{n} \geq 1$ such that $\limsup _{n \rightarrow \infty} M_{n}^{1 / n}=1$ and

$$
\left\|p e^{-n \varphi}\right\|_{Y_{r}}:=\max _{z \in Y_{r}}|p(z)| e^{-n \varphi(z)} \leq M_{n}\left\|p e^{-n \varphi}\right\|_{L^{2}(\nu)} \forall p \in \mathcal{P}_{n} .
$$

If $Y$ is an unbounded, we also require

$$
\int_{Y \backslash Y_{r}} \frac{1}{\|z\|^{a}} d \nu<\infty \text { for some } a>0 .
$$

The conditions (2.1) and (2.6) ensure that the weighted measure $e^{-2 n \varphi} d \nu$ has finite moments up to order $n$. Whereas condition (2.5) implies that $L^{2}$ and sup norms of weighted polynomials are asymptotically equivalent. We also remark that BM-measures always exist (see eg. [BLPW15]).

2.1. Domination Principle. Let $X=\mathbb{P}^{m}$ be the complex projective space and $\omega$ denotes the Fubini-Study Kähler form normalized by $\int_{X} \omega^{m}=1$. We also denote the set of all $\omega$-psh functions by

$$
P S H(X, \omega)=\left\{\phi \in L^{1}(X) \mid \phi \text { usc and } \omega+d d^{c} \phi \geq 0\right\} .
$$

Following [GZ07], we define non-pluripolar Monge-Ampére of $\phi \in P S H(X, \omega)$ by

$$
M A(\phi):=\lim _{j \rightarrow \infty}\left\{\mathbb{1}_{\{\phi>-j\}}\left(\omega+d d^{c} \max [\phi,-j]\right)^{m}\right\} .
$$

It follows from [GZ07] that the $M A(\phi)$ is a (positive) Borel measure satisfying

$$
\int_{X} M A(\phi) \leq \int_{X} \omega^{m}=1
$$

Definition 2.1. We denote

$$
\mathcal{E}(X, \omega):=\left\{\phi \in P S H(X, \omega) \mid \int_{X} M A(\phi)=1\right\} .
$$

Then we have the following generalized domination principle due to Dinew [Din09]:

Theorem 2.2. Let $\psi \in P S H(X, \omega)$ and $\phi \in \mathcal{E}(X, \omega)$ that satisfy $\psi \leq \phi$ a.e. with respect to $M A(\phi)$. Then $\psi \leq \phi$ on $X$.

It is well know that (see eg. [Dem09]) there is a 1-1 correspondence between Lelong class psh function $\mathcal{L}\left(\mathbb{C}^{m}\right)$ and the set of $\omega$-psh functions which is given by the natural identification

$$
u \in \mathcal{L}\left(\mathbb{C}^{m}\right) \rightarrow \varphi(z):= \begin{cases}u(z)-\frac{1}{2} \log \left(1+\|z\|^{2}\right) & \text { for } z \in \mathbb{C}^{m} \\ \lim \sup _{w \in \mathbb{C}^{m} \rightarrow z} u(w)-\frac{1}{2} \log \left(1+\|w\|^{2}\right) & \text { for } z \in H_{\infty}\end{cases}
$$

where $\mathbb{P}^{m}=\mathbb{C}^{m} \cup H_{\infty}$ and $H_{\infty}$ denotes the hyperplane at infinity. 
Now, writing $u \in \mathcal{L}\left(\mathbb{C}^{m}\right)$ as $u=\phi+u_{0}$ where $\phi \in P S H(X, \omega)$ and $u_{0}(z)=\frac{1}{2} \log \left(1+\|z\|^{2}\right)$ on $\mathbb{C}^{m}$ we see that

$$
N P\left(d d^{c} u\right)^{m}=M A(\phi)
$$

on $\mathbb{C}^{m}$ where

$$
N P\left(d d^{c} u\right)^{m}=\lim _{j \rightarrow \infty}\left\{\mathbb{1}_{\{u>-j\}}\left(d d^{c} \max [u,-j]\right)^{m}\right\}
$$

denotes the non-pluripolar Monge-Ampère (cf. [BT87, §4]). Hence, we obtain the following $\mathbb{C}^{m}$ version of Dinew's domination principle:

Corollary 2.3. Let $u, v \in \mathcal{L}\left(\mathbb{C}^{m}\right)$ and assume that

$$
\int_{\mathbb{C}^{m}} N P\left(d d^{c} u\right)^{m}=1 .
$$

If $v \leq u$ a.e with respect to $N P\left(d d^{c} u\right)^{m}$ then $v \leq u$ on $\mathbb{C}^{m}$.

2.2. Mass Asymptotics. We continue with a basic result which asserts that mass equidistribution for sequences of weighted polynomials implies $L_{l o c}^{1}$-convergence of potentials to the weighted global extremal function.

Theorem 2.4. Let $Y \subset \mathbb{C}^{m}$ be a locally regular closed set, $\varphi: Y \rightarrow \mathbb{R}$ be a continuous weight function and $\nu$ be a BM-measure for the weighted set $(Y, \varphi)$. If $Y$ is unbounded, we also require $\varphi(z)$ to verify (2.1). We assume that

$$
\int_{\operatorname{Int}\left(S_{Y, \varphi}\right)} d \mu_{Y, \varphi}=1
$$

Furthermore, let $p_{n} \in \mathcal{P}_{n}$ be a sequence of polynomials verifying

$$
\limsup _{n \rightarrow \infty} \frac{1}{n} \log \left\|p_{n} e^{-n \varphi}\right\|_{L^{2}(\nu)} \leq 0
$$

and assume that

$$
\frac{1}{d_{n}}\left|p_{n}(z)\right|^{2} e^{-2 n \varphi} d \nu \rightarrow \mu_{Y, \varphi}
$$

in the weak-star topology of measures on $S_{Y, \varphi}$. Then

$$
\frac{1}{n} \log \left|p_{n}\right| \rightarrow V_{Y, \varphi} \text { in } L_{l o c}^{1}\left(\mathbb{C}^{m}\right) .
$$

In particular,

in the sense of currents.

$$
\frac{1}{n}\left[Z_{p_{n}}\right]:=\frac{1}{n} d d^{c} \log \left|p_{n}\right| \rightarrow d d^{c}\left(V_{Y, \varphi}\right)
$$

The hypothesis (2.13) means that for each continuous function $u \in C\left(S_{Y, \varphi}\right)$ we have

$$
\frac{1}{d_{n}} \int_{S_{Y, \varphi}} u(z)\left|p_{n}(z)\right|^{2} e^{-2 n \varphi(z)} d \nu \rightarrow \int_{S_{Y, \varphi}} u(z) d \mu_{Y, \varphi} \text { as } n \rightarrow \infty .
$$

We remark that the normalization factor $\frac{1}{d_{n}}$ is non-standard (cf. [SZ99, Zel18]). However, the current version is more suitable for our purposes (cf. Theorem 1.1). In complex dimension one, $\left[Z_{p_{n}}\right]=\sum_{p_{n}(z)=0} \delta_{z}$ becomes counting measure on zeros of $p_{n}$. Hence, Theorem 2.4 gives a sufficient condition for zeros of weighted polynomials to be equidistributed with respect to the associated equilibrium measure. We also remark that assumption (2.11) requires, in particular, that $\operatorname{Int}\left(S_{Y, \varphi}\right)$ is a non-empty open subset of $\mathbb{C}^{m}$. This is necessary as the following example shows: 
Example 2.5. In the spacial case $Y=S^{1}$ unit circle and $\varphi \equiv 0$ we have $V_{Y}(z)=\log ^{+}|z|$ and $\mu_{Y, \varphi}=\frac{1}{2 \pi} d \theta$ is the normalized arc-length measure. In this case, the monomials $p_{n}(z)=z^{n}$ and $\nu=\frac{1}{2 \pi} d \theta$ satisfy the hypotheses of the Theorem 2.4 but $\frac{1}{n} \log \left|p_{n}(z)\right|=\log |z| \neq \log ^{+}|z|$ in $L_{l o c}^{1}(\mathbb{C})$.

We thank Tom Bloom for pointing this example out. We are also grateful to N. Levenberg for his comments on an earlier draft.

Proof of Theorem 2.4. We fix $r \gg 1$ such that $V_{Y, \varphi}=V_{Y_{r}, \varphi}$ which implies that $S_{Y, \varphi} \subset Y_{r}$. Then by (2.12) and BM inequality (2.5) for each $\epsilon>0$

$$
\left\|p_{n} e^{-n \varphi}\right\|_{S_{Y, \varphi}} \leq\left\|p_{n} e^{-n \varphi}\right\|_{Y_{r}} \leq e^{\epsilon n} M_{n}
$$

for sufficiently large $n$. It follows from Theorem 2.5 of [ST97, Appendix B], continuity of $\varphi$ and $V_{Y, \varphi}$ that $V_{Y, \varphi}=\varphi$ on $S_{Y, \varphi}$. This implies that

$$
\left|p_{n}(z)\right| \leq M_{n} e^{n\left(V_{Y, \varphi}(z)+\epsilon\right)} \forall z \in S_{Y, \varphi} .
$$

Applying [ST97, Theorem 2.6 in Appendix B] we deduce that

$$
\left|p_{n}(z)\right| \leq M_{n} e^{n\left(V_{Y, \varphi}(z)+\epsilon\right)} \forall z \in \mathbb{C}^{m}
$$

for sufficiently large $n$. Since $\epsilon>0$ arbitrary, by [Kli91, Theorem 5.2.1] we conclude that for every sequence of positive integers $J$ the function

$$
G(z):=\left(\limsup _{n \in J} \frac{1}{n} \log \left|p_{n}(z)\right|\right)^{*} \in \mathcal{L}\left(\mathbb{C}^{m}\right)
$$

and satisfies

$$
G \leq V_{Y, \varphi} \text { on } \mathbb{C}^{m}
$$

Next, we claim that

$$
G(z)=V_{Y, \varphi}(z) \text { on } \operatorname{Int}\left(S_{Y, \varphi}\right) .
$$

Postponing the proof of the claim for the moment and assuming (2.18), since $V_{Y, \varphi}$ is locally bounded on $\mathbb{C}^{m}$ and $\operatorname{Int}\left(S_{Y, \varphi}\right)$ is an open subset of $\mathbb{C}^{m}$, by (2.11) and (2.8) we deduce that

$$
\int_{\mathbb{C}^{m}} N P\left(d d^{c} G\right)^{m}=\int_{\operatorname{Int}\left(S_{Y, \varphi}\right)} N P\left(d d^{c} G\right)^{m}=\int_{\operatorname{Int}\left(S_{Y, \varphi}\right)} d \mu_{Y, \varphi}=1 .
$$

Here, $N P\left(d d^{c} G\right)^{m}$ denotes the non-pluripolar Monge-Ampère of $G$. This implies that

$$
G(z)=V_{Y, \varphi}(z) \text { a.e. with respect to } N P\left(d d^{c} G\right)^{m} \text {. }
$$

Thus, we can apply domination principle Corollary 2.3 with $u=G$ and $v=V_{Y, \varphi}$ to conclude that

$$
G=V_{Y, \varphi} \text { on } \mathbb{C}^{m} \text {. }
$$

Hence, the theorem follows from [BL15, Proposition 4.4].

Now, we return the proof of the claim (2.18). To this end, assume that $G(w)<V_{Y, \varphi}(w)$ for some $w \in \operatorname{Int}\left(S_{Y, \varphi}\right)$. We fix an open ball $w \in B \subset \operatorname{Int}\left(S_{Y, \varphi}\right)$. Note that by (2.15) and [H9̈4, Theorem 3.2.12] there are two options:

(i) $\frac{1}{n} \log \left|p_{n}\right| \rightarrow-\infty$ locally uniformly on $B$

(ii) there exists a further subsequence $J_{1}$ such that for $n \in J_{1}$

$$
\frac{1}{n} \log \left|p_{n}\right| \rightarrow g \text { in } L^{1}(B) \text {. }
$$


First, we rule out the option (i). Indeed, otherwise

$$
\left|p_{n}\right| e^{-n \varphi} \ll 1 \text { locally uniformly on } B \text { for } n \gg 1
$$

which contradicts (2.13). Thus, (ii) occurs. Then passing to a further subsequence $J_{2} \subset J_{1}$ we conclude that

$$
\frac{1}{n} \log \left|p_{n}\right| \rightarrow g \text { a.e. on } B \text {. }
$$

Note that $g^{*}$ is psh on $B$ and $g^{*}=g$ a.e on $B$ hence $g^{*} \leq G$ on $B$ which in turn implies that $g^{*}(w)<V_{Y, \varphi}(w)$. Then by Hartogs' lemma and continuity of $V_{Y, \varphi}$ there exists $\delta, \rho>0$ such that $B(w, \rho) \subset B$ and

$$
\frac{1}{n} \log \left|p_{n}(z)\right|<V_{Y, \varphi}(z)-\delta, \forall z \in B(w, \rho)
$$

for large $n \in J_{2}$. Since $V_{Y, \varphi} \leq \varphi$ on $Y$ we infer that

$$
\left|p_{n}(z)\right| e^{-n \varphi(z)} \leq e^{-n \delta} \forall z \in B(w, \rho)
$$

for large $n \in J_{2}$. This contradicts (2.13). Hence, we conclude that

$$
g^{*}(w)=V_{Y, \varphi}(w) \leq G(w) \text { for } w \in \operatorname{Int}\left(S_{Y, \varphi}\right) .
$$

This finishes the proof.

\section{Mass Asymptotics of RANDom Polynomials}

In the rest of this paper we consider the special case where $Y=\mathbb{C}^{m}$ and $\varphi: \mathbb{C}^{m} \rightarrow \mathbb{R}$ is a $\mathscr{C}^{1,1}$ function. We also assume that $\varphi$ verifies (1.1). We denote the corresponding global extremal function

$$
\varphi_{e}(z):=V_{\mathbb{C}^{m}, \varphi}(z)=\sup \left\{\psi(z): \psi \in \mathcal{L}\left(\mathbb{C}^{m}\right), \psi \leq \varphi \text { on } \mathbb{C}^{m}\right\}
$$

and the support $S_{\varphi}:=S_{\mathbb{C}^{m}, \varphi}$ of the Monge-Ampère $\mu_{\varphi_{e}}:=\frac{1}{m !}\left(d d^{c} V_{\mathbb{C}^{m}, \varphi}\right)^{m}$.

In [Ber09a, Corollary 3.6] Berman proved that

$$
S_{\varphi}:=\left\{z \in \mathbb{C}^{m}: \varphi(z)=\varphi_{e}(z) \text { and } d d^{c} \varphi(z)>0\right\}
$$

and

$$
\mu_{\varphi_{e}}=\mathbb{1}_{S_{\varphi}} \operatorname{det}\left(d d^{c} \varphi\right) d V_{m} .
$$

We remark that by $\mathscr{C}^{1,1}$ regularity $d d^{c} \varphi(z)=\frac{i}{\pi} \sum_{j, k} \frac{\partial^{2} \varphi}{\partial z_{j} \partial \bar{z}_{k}} d z_{j} \wedge d \bar{z}_{k}$ is well-defined at Lebesgue almost every $z \in \mathbb{C}^{m}$ and the condition $d d^{c} \varphi(z)>0$ implies all eigenvalues of the Hessian $\left[\frac{\partial^{2} \varphi}{\partial z_{j} \partial \bar{z}_{k}}\right]_{j, k}$ are positive. Moreover, $\operatorname{det}\left(d d^{c} \varphi\right):=\left(\frac{2}{\pi}\right)^{m} \operatorname{det}\left[\frac{\partial^{2} \varphi}{\partial z_{j} \partial \bar{z}_{k}}\right]$.

3.1. Bergman Kernel Asymptotics. For a fixed orthonormal basis (ONB) $\left\{P_{j}^{n}\right\}_{j=1}^{d_{n}}$ for $\mathcal{P}_{n}$ with respect to the norm (1.2) the Bergman kernel is given by

$$
K_{n}(z, w):=\sum_{j=1}^{d_{n}} P_{j}^{n}(z) \overline{P_{j}^{n}(w)} .
$$

We also denote the Bergman function by

$$
B_{n}(z):=K_{n}(z, z) e^{-2 n \varphi(z)}=\sum_{j=1}^{d_{n}}\left|P_{j}^{n}(z)\right|^{2} e^{-2 n \varphi(z)} .
$$


Bergman function $B_{n}$ has the extremal property

$$
B_{n}(z)=\sup _{f_{n} \in \mathcal{P}_{n} \backslash\{0\}} \frac{\left|f_{n}(z)\right|^{2} e^{-2 n \varphi(z)}}{\left\|f_{n}\right\|_{n}^{2}} .
$$

where $\left\|f_{n}\right\|_{n}$ denotes the norm induced by (1.2). Moreover, we have the following dimensional density property

$$
\int_{\mathbb{C}^{m}} B_{n}(z) d V_{m}(z)=\operatorname{dim}\left(\mathcal{P}_{n}\right)=O\left(n^{m}\right)
$$

The following result will be useful in order to obtain expected mass distribution of random polynomials (see Proposition 3.8).

Theorem 3.1. [Ber09b] Let $\varphi: \mathbb{C}^{m} \rightarrow \mathbb{R}$ be a $\mathscr{C}^{1,1}$-weight function satisfying (1.1). Then

$$
n^{-m} K_{n}(z, z) e^{-2 n \varphi(z)} \rightarrow \mathbb{1}_{S_{\varphi}} \operatorname{det}\left(d d^{c} \varphi\right)
$$

in $L^{1}\left(\mathbb{C}^{m}\right)$. In particular, $n^{-m} K_{n}(z, z) e^{-2 n \varphi(z)} d V_{m}(z)$ converges to the weighted equilibrium measure $\mu_{\varphi_{e}}$ in the weak-star topology on $\mathbb{C}^{m}$.

The next result is also due to Berman [Ber09b, Theorem 3.8] which allows us to get asymptotic Hilbert-Schmidt norms of the Toeplitz operators (see Proposition 3.3):

Theorem 3.2. Let $\varphi: \mathbb{C}^{m} \rightarrow \mathbb{R}$ be a $\mathscr{C}^{1,1}$-weight function satisfying (1.1). Then

$$
n^{-m}\left|K_{n}(z, w)\right|^{2} e^{-2 n \varphi(z)-2 n \varphi(w)} d V_{m}(z) d V_{m}(w) \rightarrow \Delta \wedge \mathbb{1}_{S_{\varphi}} \mu_{\varphi_{e}}
$$

as measures on $\mathbb{C}^{m} \times \mathbb{C}^{m}$ in weak-star topology.

Here; $\Delta:=[\{z=w\}]$ denotes the current of integration along the diagonal in $\mathbb{C}^{m} \times \mathbb{C}^{m}$ and for any bounded continuous function $\Psi$ we have

$$
\int_{\mathbb{C}^{m} \times \mathbb{C}^{m}} \Psi(x, y) \Delta \wedge \mathbb{1}_{S_{\varphi}} \mu_{\varphi_{e}}:=\int_{S_{\varphi}} \Psi(x, x) d \mu_{\varphi_{e}} .
$$

3.2. Toeplitz operators and limiting distribution of eigenvalues. We denote the orthogonal projection

$$
\Pi_{n}: L^{2}\left(\mathbb{C}^{m}, e^{-2 n \varphi(z)} d V_{m}\right) \rightarrow \mathcal{P}_{n}
$$

onto the finite dimensional subspace $\mathcal{P}_{n}$. For a bounded function $g: \mathbb{C}^{m} \rightarrow \mathbb{R}$ we also let

$$
M_{g}: L^{2}\left(\mathbb{C}^{m}, e^{-2 n \varphi(z)} d V_{m}\right) \rightarrow L^{2}\left(\mathbb{C}^{m}, e^{-2 n \varphi(z)} d V_{m}\right)
$$

denote multiplication operator defined by

$$
M_{g}(h)(z)=g(z) h(z) .
$$

We consider the sesquilinear form on $\mathcal{P}_{n}$ defined by

$$
\langle p, q\rangle_{g}:=\int_{\mathbb{C}^{m}} g(z) p(z) \overline{q(z)} e^{-2 n \varphi(z)} d V_{m} .
$$

Then by linear algebra there is a self-adjoint operator $T_{n}^{g}: \mathcal{P}_{n} \rightarrow \mathcal{P}_{n}$ such that

$$
\langle p, q\rangle_{g}=\left\langle T_{n}^{g} p, q\right\rangle_{n} .
$$

Note that $T_{n}^{g} p$ is nothing but the composition of orthogonal projection with the multiplication operator on $\mathcal{P}_{n}$ i.e.

$$
T_{n}^{g}=\Pi_{n} \circ M_{g}
$$


which is called $n^{\text {th }}$ Toeplitz operator with multiplier $g$. The latter property implies that

$$
T_{n}^{g} p(z)=\int_{\mathbb{C}^{m}} g(w) p(w) K_{n}(z, w) e^{-2 n \varphi(w)} d V_{m}(w) .
$$

The following is a standard result in this setting and it indicates a connection between the Toeplitz operators and mass equidistribution:

Proposition 3.3. Let $\varphi: \mathbb{C}^{m} \rightarrow \mathbb{R}$ be a $\mathscr{C}^{1,1}$-weight function satisfying (1.1) and $g: \mathbb{C}^{m} \rightarrow \mathbb{R}$ be a bounded function. Then

(1) $\operatorname{Tr}\left(T_{n}^{g}\right)=\int_{\mathbb{C}^{m}} g(z) K_{n}(z, z) e^{-2 n \varphi(z)} d V_{m}$.

(2) For each $k \in \mathbb{N}$ we have

$$
\frac{1}{d_{n}} \operatorname{Tr}\left(\left(T_{n}^{g}\right)^{k}\right) \rightarrow \int_{\mathbb{C}^{m}} g^{k}(z) d \mu_{\varphi_{e}}
$$

as $n \rightarrow \infty$.

Proof. (1) Note that $T_{n}^{g}$ admits an ONB of eigenvectors $\left\{p_{j}^{n}\right\}_{j=1}^{d_{n}}$. Letting

$$
\mu_{j}:=\left\langle T_{n}^{g} p_{j}^{n}, p_{j}^{n}\right\rangle_{n}=\left\langle p_{j}^{n}, p_{j}^{n}\right\rangle_{g}
$$

we obtain

$$
\operatorname{Tr}\left(T_{n}^{g}\right)=\sum_{j=1}^{d_{n}} \int_{\mathbb{C}^{m}} g(z)\left|p_{j}^{n}(z)\right|^{2} e^{-2 n \varphi(z)} d V_{m}=\int_{\mathbb{C}^{m}} g(z) K_{n}(z, z) e^{-2 n \varphi(z)} d V_{m} .
$$

(2) It follows from Theorem 3.1 that

$$
\frac{1}{d_{n}} \operatorname{Tr}\left(T_{n}^{g}\right) \rightarrow \int_{\mathbb{C}^{m}} g(z) d \mu_{\varphi_{e}} .
$$

Note that $\left(T_{n}^{g}\right)^{2}=\Pi_{n} M_{g} \Pi_{n} M_{g}$ and

$$
\operatorname{Tr}\left(\left(T_{n}^{g}\right)^{2}\right)=\int_{\mathbb{C}^{m}} \int_{\mathbb{C}^{m}} g(z) g(w)\left|K_{n}(z, w)\right|^{2} e^{-2 n(\varphi(z)+\varphi(w))} d V_{m}(z) d V_{m}(w) .
$$

Hence, by Theorem 3.2 we have

$$
\frac{1}{d_{n}} \operatorname{Tr}\left(\left(T_{n}^{g}\right)^{2}\right) \rightarrow \int_{\mathbb{C}^{m}} \int_{\mathbb{C}^{m}} g(z) g(w) \Delta \wedge \mathbb{1}_{S_{\varphi}} \mu_{\varphi_{e}}=\int_{\mathbb{C}^{m}} g^{2}(z) d \mu_{\varphi_{e}} .
$$

Now, for $k \geq 3$ we have

$$
\mu_{j}^{k}=\left\langle\left(\Pi_{n} M_{g}\right)^{k} p_{j}^{n}, p_{j}^{n}\right\rangle_{n}
$$

and hence,

$$
\sum_{j=1}^{d_{n}} \mu_{j}^{k}=\int_{\mathbb{C}^{m}} \int_{\mathbb{C}^{m}} g(z) g(w)^{k-1}\left|K_{n}(z, w)\right|^{2} e^{-2 n \varphi(z)-2 n \varphi(w)} d V_{m}(z) d V_{m}(w) .
$$

Thus, it follows from Theorem 3.2 that

$$
\frac{1}{d_{n}} \operatorname{Tr}\left(\left(T_{n}^{g}\right)^{k}\right) \rightarrow \int_{\mathbb{C}^{m}} g^{k}(z) d \mu_{\varphi_{e}}
$$


3.3. Subgaussian Random Variables. In this section we recall basic properties of subgaussian random variables. Let $(\Omega, \mathcal{F}, \tau)$ be a probability space. A real valued random variable $X: \Omega \rightarrow \mathbb{R}$ is called subgaussian with parameter $b>0$ (or $b$-subgaussian) if the moment generating function (MGF) of $X$ is dominated by MGF of normalized Gaussian $N(0, b)$ that is

$$
\mathbb{E}\left[e^{t X}\right] \leq e^{\frac{b^{2} t^{2}}{2}} \text { for all } t \in \mathbb{R} .
$$

We remark that the above definition is non-standard (cf. [Ver12, §5.2.3]); in particular (3.6) forces that $\mathbb{E}[X]=0$ which is a convenient assumption for our setting. The classical examples of 1subgaussian random variables are Standard Gaussian $N(0,1)$, Bernoulli random variables $\mathbb{P}[X=$ $\pm 1]=\frac{1}{2}$, and uniform distribution on $[-1,1]$. Moreover, all bounded random variables of mean zero are subgaussian. More precisely, if $\mathbb{E}[X]=0$ and $X \leq b$ almost surely then $X$ is $b$-subgaussian. We have the following characterization of subgaussian random variables.

Proposition 3.4. [Ver12, Lemma 5.5] Let $X$ be a centered real random variable (i.e. $\mathbb{E}[X]=0$ ). Then the following are equivalent:

(1) $\exists b>0$ such that $\mathbb{E}\left[e^{t X}\right] \leq e^{\frac{b^{2} t^{2}}{2}}$ for all $t \in \mathbb{R}$.

(2) $\exists c>0$ such that $\mathbb{P}[|X|>\alpha] \leq 2 e^{-c \alpha^{2}}$ for every $\alpha>0$.

(3) $\exists K>0$ such that $\left(\mathbb{E}\left[|X|^{p}\right)^{\frac{1}{p}} \leq K \sqrt{p}\right.$ for all $p \geq 1$.

(4) $\exists \kappa>0$ such that $\mathbb{E}\left[e^{X^{2} / \kappa^{2}}\right] \leq 2$.

The last property is known as $\psi_{2}$ condition. More precisely, a centered random variable $X$ is subgaussian if and only if its Orlicz norm

$$
\|X\|_{\psi_{2}}:=\inf _{\kappa>0}\left\{\mathbb{E}\left[e^{X^{2} / \kappa^{2}}\right] \leq 2\right\}
$$

is finite.

3.3.1. Hanson-Wright Inequality. Let $X_{j}$ be independent subgaussian random variables and $\kappa_{j}:=$ $\left\|X_{j}\right\|_{\psi_{2}}$. We denote the joint probability distribution of $X:=\left(X_{1}, \ldots, X_{N}\right)$ by $\mathbb{P}$. We also let $A=\left[A_{i j}\right]$ be a square matrix with real entries. We denote its operator norm

$$
\|A\|:=\max _{\|v\|_{2} \leq 1}\|A v\|
$$

where $\|\cdot\|_{2}$ denotes Euclidean norm and the Hilbert-Schmidt norm by

$$
\|A\|_{H S}:=\left(\sum_{i, j}\left|a_{i j}\right|^{2}\right)^{1 / 2}=\left[\operatorname{Tr}\left(A A^{T}\right)\right]^{1 / 2} .
$$

We consider the random quadratic form

$$
X \rightarrow X^{T} A X .
$$

The following concentration inequality goes back to Hanson-Wright [HW71]. The version we use here is due to Rudelson-Vershynin [RV13]:

Theorem 3.5 (Hanson-Wright Inequality). Let $A$ be a $N \times N$ square matrix and $X=\left(X_{1}, \ldots, X_{N}\right) \in$ $\mathbb{R}^{N}$ be a random vector whose components $X_{j}$ are independent subgaussian variables such that

for $j=1, \ldots, N$. Then for each $t \geq 0$

$$
\left\|X_{j}\right\|_{\psi_{2}} \leq K
$$

$$
\mathbb{P}\left[\left|X^{T} A X-\mathbb{E}\left[X^{T} A X\right]\right|>t\right] \leq 2 \exp \left(-c \min \left\{\frac{t^{2}}{K^{4}\|A\|_{H S}^{2}}, \frac{t}{K^{2}\|A\|}\right\}\right)
$$

where $c>0$ is an absolute constant which does not depend on $t$. 
3.3.2. Complex Case. Next, we formulate Hanson-Wright inequality for complex random variables and Hermitian matrices with complex entries. Let $X: \Omega \rightarrow \mathbb{C}$ be a complex valued random variable. We denote the real and imaginary parts of $X$ by $\operatorname{Re}(X)$ and $\operatorname{Im}(X)$ respectively. We say that $X$ is subgaussian if $\operatorname{Re}(X)$ and $\operatorname{Im}(X)$ are independent subgaussian random variables. For a Hermitian square matrix $A$ we let

$$
\tilde{A}=\left[\begin{array}{cc}
\operatorname{Re}(A) & -\operatorname{Im}(A) \\
\operatorname{Im}(A) & \operatorname{Re}(A)
\end{array}\right]
$$

where $\operatorname{Re}(A):=\left[\operatorname{Re}\left(a_{i j}\right)\right]$ and $\operatorname{Im}(A)=\left[\operatorname{Im}\left(a_{i j}\right)\right]$. Under these definitions we have $\|\tilde{A}\|=\|A\|$ and $\|\tilde{A}\|_{H S}=\sqrt{2}\|A\|_{H S}$. We also let $X^{*}:=\bar{X}^{T}$. Then applying Theorem 3.5 we obtain:

Theorem 3.6 (Complex Hanson Wright Inequality). Let $A$ be a $N \times N$ Hermitian matrix and $X=$ $\left(X_{1}, \ldots, X_{N}\right) \in \mathbb{C}^{N}$ be a random vector whose components $X_{j}$ are independent complex subgaussian random variables such that $\max _{j}\left(\left\|\operatorname{Re}\left(X_{j}\right)\right\|_{\psi_{2}},\left\|\operatorname{Im}\left(X_{j}\right)\right\|_{\psi_{2}}\right) \leq K$. Then there exists an absolute constant $c>0$ such that

$$
\mathbb{P}\left[\left|X^{*} A X-\mathbb{E}\left[X^{*} A X\right]\right|>t\right] \leq 2 \exp \left(-c \min \left\{\frac{t^{2}}{K^{4}\|A\|_{H S}^{2}}, \frac{t}{K^{2}\|A\|}\right\}\right)
$$

for each $t \geq 0$.

Remark 3.7. Finally, we remark that in case $A$ is Hermitian matrix and $X_{j}$ are real valued subgaussian by setting $\tilde{A}:=\left[\begin{array}{l}\operatorname{Re}(A) \\ \operatorname{Im}(A)\end{array}\right]$ the corresponding concentration inequality follows from $[\mathrm{RV} 13$, Theorem 2.1].

3.4. Mass Equidistribution for Random Polynomials. For a fixed ONB $\left\{P_{j}^{n}\right\}_{j=1}^{d_{n}}$ of $\mathcal{P}_{n}$ with respect to the norm induced by (1.2) we consider subgaussian random polynomials

$$
f_{n}(z)=\sum_{j=1}^{d_{n}} c_{j}^{n} P_{j}^{n}(z)
$$

where $c_{j}^{n}$ are independent identically distributed (iid) real or complex subgaussian random variables of mean zero and unit variance i.e. $\mathbb{E}\left[\left|c_{j}^{n}\right|^{2}\right]=1$. We endow the vector space $\mathcal{P}_{n}$ with the $d_{n}$-fold product probability measure $\mathrm{Prob}_{n}$ induced by the law of $c_{j}^{n}$. We also consider the product probability space $\prod_{n=1}^{\infty}\left(\mathcal{P}_{n}, \operatorname{Prob}_{n}\right)$ whose elements are sequences $\left(f_{1}, f_{2}, \ldots\right)$ of random polynomials.

Let $g: \mathbb{C}^{m} \rightarrow \mathbb{R}$ be a bounded function, in what follows we consider the random variables

$$
\begin{aligned}
& X_{n}^{g}: \mathcal{P}_{n} \rightarrow \mathbb{R} \\
X_{n}^{g}\left(f_{n}\right)= & \int_{\mathbb{C}^{m}} g(z)\left|f_{n}(z)\right|^{2} e^{-2 n \varphi(z)} d V_{m} \\
= & \left\langle T_{n}^{g}\left(f_{n}\right), f_{n}\right\rangle_{n} .
\end{aligned}
$$

Next, we obtain asymptotic expected value of $X_{n}^{g}$ :

Proposition 3.8. Assume that random coefficients $c_{j}^{n}$ are iid real or complex subgaussian random variables of mean zero and unit variance. Then

$$
\mathbb{E}\left[X_{n}^{g}\right]=\operatorname{Tr}\left(T_{n}^{g}\right) .
$$

In particular,

$$
\frac{1}{d_{n}} \mathbb{E}\left[X_{n}^{g}\right] \rightarrow \int_{\mathbb{C}^{m}} g(z) d \mu_{\varphi_{e}} \text { as } n \rightarrow \infty
$$


Proof. Note that

$$
\left|f_{n}(z)\right|^{2}=\sum_{1 \leq j, k \leq d_{n}} c_{j} \overline{c_{k}} P_{j}(z) \overline{P_{k}(z)}
$$

Since $c_{j}^{n}$ are iid of mean zero and unit variance, we have $\mathbb{E}\left[\left|f_{n}(z)\right|^{2}\right]=K_{n}(z, z)$ for every $z \in \mathbb{C}^{m}$. Thus, by Fubini's Theorem

$$
\mathbb{E}\left[X_{n}^{g}\right]=\int_{\mathbb{C}^{m}} g(z) K_{n}(z, z) e^{-2 n \varphi(z)} d V_{m}=\operatorname{Tr}\left(T_{n}^{g}\right) .
$$

Hence, the second assertion follows from Proposition 3.3.

Proof of Theorem 1.1. We prove the case where $c_{j}^{n}$ are iid complex subgaussian random variables. The real case follows from the same argument and Remark 3.7.

For the fixed ONB basis of eigenfunctions $\left\{P_{j}^{n}\right\}_{j=1}^{d_{n}}$ we may identify the random polynomials

$$
f_{n}=\sum_{j=1}^{d_{n}} c_{j}^{n} P_{j}^{n}
$$

with the subgaussian random vector

$$
Z_{n}:=\left(c_{1}^{n}, \ldots, c_{d_{n}}^{n}\right) \in \mathbb{C}^{d_{n}}
$$

and denote the probability law of $Z_{n}$ by $\mathbb{P}_{n}$. We also denote Euclidean norm of $Z_{n}$ by $\left\|Z_{n}\right\|$. First, we observe that

$$
\mathbb{P}_{n}\left[\left\{Z_{n} \in \mathbb{C}^{d_{n}}:\left\|Z_{n}\right\| \leq d_{n} \text { for sufficiently large } n\right\}\right]=1 .
$$

Indeed, by Proposition 3.4 (2) there exists $b>0$ (independent of $n$ ) such that

$$
\begin{aligned}
\mathbb{P}_{n}\left[\left\{Z_{n} \in \mathbb{C}^{d_{n}}:\left\|Z_{n}\right\|>d_{n}\right\}\right] & =\mathbb{P}_{n}\left[\left\{c_{j}^{n} \in \mathbb{C}^{d_{n}}: \sum_{j=1}^{d_{n}}\left|c_{j}^{n}\right|^{2}>d_{n}^{2}\right\}\right] \\
& \leq \mathbb{P}_{n}\left[\left\{c_{j}^{n} \in \mathbb{C}^{d_{n}}:\left|c_{j}^{n}\right|^{2}>d_{n} \text { for some } j\right\}\right] \\
& \leq 2 d_{n} \exp \left(-b d_{n}\right) .
\end{aligned}
$$

Since the right hand side of the last inequality (3.11) is summable over $n$, the claim follows from Borel-Cantelli lemma.

Next, we identify the operator $T_{n}^{g}$ with a Hermitian $d_{n} \times d_{n}$ matrix $A_{n}^{g}$. Note that with the new notation we have

$$
X_{n}^{g}\left(f_{n}\right)=\left\langle A_{n}^{g} Z_{n}, Z_{n}\right\rangle
$$

where $\langle$,$\rangle denotes the standard Hermitian inner product on \mathbb{C}^{d_{n}}$. Then by Theorem 3.6 there exists an absolute constant $c>0$ such that

$$
\begin{aligned}
\operatorname{Prob}_{n}\left[\left|X_{n}^{g}\left(f_{n}\right)-\mathbb{E}\left[X_{n}^{g}\right]\right|>t\right] & =\mathbb{P}_{n}\left[\left|Z_{n}^{*} A_{n}^{g} Z_{n}-\mathbb{E}\left[Z_{n}^{*} A_{n}^{g} Z_{n}\right]\right|>t\right] \\
& \leq 2 \exp \left(-c \min \left\{\frac{t^{2}}{K^{4}\left\|A_{n}^{g}\right\|_{H S}^{2}}, \frac{t}{K^{2}\left\|A_{n}^{g}\right\|}\right\}\right)
\end{aligned}
$$

where $K:=\left\|c_{j}^{n}\right\|_{\psi_{2}} \geq 1$. Note that $\left\|A_{n}^{g}\right\| \leq \sup _{z \in \mathbb{C}^{m}}|g(z)|$ and by Proposition 3.3 we have

$$
\left\|A_{n}^{g}\right\|_{H S}^{2}=\operatorname{Tr}\left(\left(T_{n}^{g}\right)^{2}\right)=O\left(d_{n}\right) .
$$

Letting $t=\epsilon d_{n}$, by Theorem 3.1 we deduce that for sufficiently large $n$

$$
\operatorname{Prob}_{n}\left[\left|\frac{1}{d_{n}} X_{n}^{g}\left(f_{n}\right)-\int_{\mathbb{C}^{m}} g(z) d \mu_{\varphi_{e}}\right|>\epsilon\right] \leq 2 \exp \left(-C_{g} d_{n}\right)
$$


where $C_{g}>0$ is an absolute constant that deos not depend on $n$. Hence, it follows from BorelCantelli lemma that there exists a set $\mathcal{A}_{g} \subset \prod_{n=1}^{\infty} \mathcal{P}_{n}$ of probability one such that

$$
\frac{1}{d_{n}} X_{n}^{g}\left(f_{n}\right) \rightarrow \int g d \mu_{\varphi_{e}}
$$

for every $\left\{f_{n}\right\} \in \mathcal{A}_{g}$. Next, we let $\left\{g_{j}\right\}_{j=1}^{\infty}$ be a countable dense subset of $C\left(S_{\varphi}\right)$ and define

$$
\mathcal{A}:=\cap_{j=1}^{\infty} \mathcal{A}_{g_{j}} \cap\left\{\left(f_{n}\right) \in \prod_{n=1}^{\infty} \mathcal{P}_{n}:\left\|p_{n}\right\|_{n}^{2} \leq d_{n} \text { for sufficiently large } n\right\} .
$$

By (3.10) and being countable intersection of sets with probability one, $\operatorname{Prob}(\mathcal{A})=1$. Moreover, for each $\left\{f_{n}\right\} \in \mathcal{A}$ we have

$$
\frac{1}{d_{n}}\left|f_{n}(z)\right|^{2} e^{-2 n \varphi(z)} d V_{m} \rightarrow d \mu_{\varphi_{e}}
$$

as measures on $S_{\varphi}$. Indeed, for each $u \in C\left(S_{\varphi}\right)$ and $\epsilon>0$ choose $g_{j}$ such that $\left\|u-g_{j}\right\|_{S_{\varphi}} \leq \epsilon$. Then for sufficiently large $n$ we have

$$
\left|\frac{1}{d_{n}} X_{n}^{g_{j}}\left(f_{n}\right)-\int g_{j} d \mu_{\varphi_{e}}\right| \leq \epsilon
$$

hence,

$$
\begin{aligned}
\left.\left|\frac{1}{d_{n}} \int_{S_{\varphi}} u(z)\right| f_{n}(z)\right|^{2} e^{-2 n \varphi(z)} d V_{m} & -\int_{S_{\varphi}} u(z) d \mu_{\varphi_{e}} \mid \\
& \leq\left\|u-g_{j}\right\|_{S_{\varphi}}\left(\frac{1}{d_{n}}\left\|f_{n}\right\|_{n}^{2}+1\right)+\left|\frac{1}{d_{n}} X_{n}^{g_{j}}\left(f_{n}\right)-\int g_{j} d \mu_{\varphi_{e}}\right| \\
& \leq 3 \epsilon .
\end{aligned}
$$

This proves the first assertion.

Since the hypotheses of Theorem 2.4 hold with probability one we obtain the second assertion.

\section{RANDOM ORTHONORMAl BASES AND Distribution OF ZERos}

In this section, we consider random orthogonal polynomials. More precisely, for a fixed ONB $\left\{P_{j}^{n}\right\}_{j=1}^{d_{n}}$ for $\mathcal{P}_{n}$ with respect to the norm (1.2) we may identify each ONB $\mathcal{B}=\left\{F_{j}^{n}\right\}_{j=1}^{d_{n}}$ for $\mathcal{P}_{n}$ with a unitary matrix $U_{\mathcal{B}} \in \mathcal{U}\left(d_{n}\right)$. Thus, we consider the set of of all orthonormal bases for $\mathcal{P}_{n}$ as a probability space by identifying it with the unitary ensemble $\mathcal{U}\left(d_{n}\right)$ endowed with the Haar probability measure. Moreover, we let $\mathcal{O N B}:=\prod_{n \geq 1} \mathcal{U}\left(d_{n}\right)$ be the product probability space. In this context, we have the following result (cf. [SZ99, Theorem 1.2] see also [Zel18]):

Theorem 4.1. For almost every sequence of $O N B \mathcal{B}=\left\{F_{j}^{n}\right\}$ in $\mathcal{O N B}$ there exists a subsequence $\Lambda_{n} \subset\left\{1, \ldots, d_{n}\right\}$ of density one (i.e. $\frac{\# \Lambda_{n}}{d_{n}} \rightarrow 1$ as $n \rightarrow \infty$ ) such that

$$
\lim _{\substack{n \rightarrow \infty \\ j \in \Lambda_{n}}} \int_{\mathbb{C}^{m}} g(z)\left|F_{j}^{n}(z)\right|^{2} e^{-2 n \varphi(z)} d V_{m}=\int_{\mathbb{C}^{m}} g(z) d \mu_{\varphi_{e}}
$$

for every bounded continuous function $g: \mathbb{C}^{m} \rightarrow \mathbb{R}$. If $m \geq 2$ then the entire sequence has this property. 
Proof. We consider the random variables

$$
\begin{gathered}
\mathcal{Y}_{n}^{g}: \mathcal{O N B} \rightarrow \mathbb{R} \\
\mathcal{Y}_{n}^{g}(\mathcal{B}):=\sum_{j=1}^{d_{n}}\left|\left\langle U_{\mathcal{B}}^{*} A_{n}^{g} U_{\mathcal{B}} e_{j}, e_{j}\right\rangle\right|^{2}
\end{gathered}
$$

where $A_{n}^{g}$ is the matrix representing the Toeplitz operator $T_{n}^{g}$ with symbol $g$ and $e_{j}$ is the standard basis element whose $j^{\text {th }}$ coordinate is 1. By (3.5) and invariance of Haar measure under leftmultiplication with a unitary matrix

$$
\begin{aligned}
\mathbb{E}\left[\left\langle U_{\mathcal{B}}^{*} A_{n}^{g} U_{\mathcal{B}} e_{j}, e_{j}\right\rangle\right] & =\int_{\mathcal{U}\left(d_{n}\right)}\left(\left\langle U^{*} A_{n}^{g} U e_{j}, e_{j}\right\rangle\right) d U \\
& =\sum_{j=1}^{d_{j}} \mu_{j} \mathbb{E}\left[\left|U_{1 j}\right|^{2}\right] \\
& =\frac{1}{d_{n}} \operatorname{Tr}\left(A_{n}^{g}\right) .
\end{aligned}
$$

Next, we consider the standardized random variables

$$
\begin{aligned}
\overline{\mathcal{Y}}_{n}^{g}(\mathcal{B}): & =\sum_{j=1}^{d_{n}}\left|\left\langle U_{\mathcal{B}}^{*} A_{n}^{g} U_{\mathcal{B}} e_{j}, e_{j}\right\rangle-\frac{1}{d_{n}} \operatorname{Tr}\left(T_{n}^{g}\right)\right|^{2} \\
& =\sum_{j=1}^{d_{n}}\left|\left\langle U_{\mathcal{B}}^{*} \tilde{A}_{n}^{g} U_{\mathcal{B}} e_{j}, e_{j}\right\rangle\right|^{2}
\end{aligned}
$$

where $\tilde{A}_{n}^{g}=A_{n}^{g}-\frac{1}{d_{n}} \operatorname{Tr}\left(T_{n}^{g}\right) I_{n}$ is of trace zero.

Then by [Zel18, Lemma 5.1] we obtain

$$
\begin{aligned}
\mathbb{E}\left[\overline{\mathcal{Y}}_{n}^{g}\right] & =\sum_{j=1}^{d_{n}} \mathbb{E}\left[\left|\left\langle U_{\mathcal{B}}^{*} \tilde{A}_{n}^{g} U_{\mathcal{B}} e_{j}, e_{j}\right\rangle\right|^{2}\right] \\
& =\int_{\mathbb{C}^{m}} g^{2} d \mu_{\varphi_{e}}-\left(\int_{\mathbb{C}^{m}} g d \mu_{\varphi_{e}}\right)^{2}+o(1) \text { as } n \rightarrow \infty .
\end{aligned}
$$

This implies that

$$
\lim _{N \rightarrow \infty} \frac{1}{N} \sum_{n=1}^{N} \mathbb{E}\left[\frac{1}{d_{n}} \overline{\mathcal{Y}}_{n}^{g}\right] \rightarrow 0
$$

since $\frac{1}{N} \sum_{n=1}^{N} \frac{1}{d_{n}} \rightarrow 0$ as $N \rightarrow \infty$.

On the other hand, since $g$ is bounded continuous function we have

$$
\left|\left\langle U_{\mathcal{B}}^{*} A_{n}^{g} U_{\mathcal{B}} e_{j}, e_{j}\right\rangle\right|=\left.\left|\int_{\mathbb{C}^{m}} g\right| P_{j}^{n}\right|^{2} e^{-2 n \varphi} d V_{m}\left|\leq \sup _{\mathbb{C}^{m}}\right| g \mid
$$

which implies that

$$
\operatorname{Var}\left[\frac{1}{d_{n}} \overline{\mathcal{Y}}_{n}^{g}\right] \leq \sup _{\mathcal{B}}\left(\frac{1}{d_{n}} \overline{\mathcal{Y}}_{n}^{g}(\mathcal{B})\right)^{2}=O(1)
$$


where the implied constant depends on $g$ but independent of $n$. Since $\frac{1}{d_{n}} \overline{\mathcal{Y}}_{n}^{g}$ are independent random variables whose variances are bounded it follows from (4.2) and Kolmogorov's law of large numbers that as $N \rightarrow \infty$

$$
\frac{1}{N} \sum_{n=1}^{N} \frac{1}{d_{n}} \bar{Y}_{n}^{g} \rightarrow 0
$$

almost surely. Thus, the first assertion follows from [Wal82, Theorem 1.20].

For the second assertion, note that for $m \geq 2$ we have $\mathbb{E}\left[\frac{1}{d_{n}} \overline{\mathcal{Y}}_{n}^{g}\right]=O\left(\frac{1}{n^{m}}\right)$ which in turn implies that $\mathbb{E}\left[\sum_{n=1}^{\infty} \frac{1}{d_{n}} \overline{\mathcal{Y}}_{n}^{g}\right]<\infty$ and hence $\frac{1}{d_{n}} \overline{\mathcal{Y}}_{n}^{g} \rightarrow 0$ almost surely.

In the unweighted case [Blo01] T. Bloom proved that for every regular compact set $K \subset \mathbb{C}^{m}$ and Bernstein-Markov measure $\nu$, every ONB $\mathcal{B}=\left\{F_{j}^{n}\right\} \in \mathcal{O N B}$ has the property that

$$
V_{K}(z)=\left(\limsup _{\substack{n \rightarrow \infty \\ j \in\left\{1, \ldots, d_{n}\right\}}} \frac{1}{n} \log \left|F_{j}^{n}(z)\right|\right)^{*} \text { for all } z \in \mathbb{C}^{m} \backslash \hat{K}
$$

where $\hat{K}$ denotes the polynomial convex hull of $K$. On the other hand, by the proof of Theorem 2.4 an immediate consequence of Theorem 4.1 is that for almost every ONB $\mathcal{B}=\left\{F_{j}^{n}\right\} \in \mathcal{O N} \mathcal{B}$

$$
\varphi_{e}(z)=\left(\limsup _{\substack{n \rightarrow \Lambda_{n} \\ j \in \Lambda_{n}}} \frac{1}{n} \log \left|F_{j}^{n}(z)\right|\right)^{*} \text { for all } z \in \mathbb{C}^{m} .
$$

However, we remark that Theorem 4.1 is a probabilistic result and the set of ONB which do not fall in its context is non-empty. For example in dimension one, for $\varphi(z)=\frac{|z|^{2}}{2}$ the $F_{j}(z)=\sqrt{\frac{n^{j+1}}{\pi j !}} z^{j}$ form an ONB for $\mathcal{P}_{n}$ with respect to the norm $\|\cdot\|_{n}$. However, zeros of $F_{j}$ are not equidistributed with respect to the equilibrium measure.

\section{FURTHER GENERALIZATIONS}

In the last part of this work we describe a generalization of Theorem 1.1 to the line bundle setting. Let $M$ be a compact complex projective Hermitian manifold and $L \rightarrow M$ be an ample holomorphic line bundle endowed with a smooth (at least $\mathscr{C}^{2}$ ) Hermitian metric $h=e^{-\varphi}$ where $\varphi=\left\{\varphi_{\alpha}\right\}$ is a local weight of the metric. The latter means that if $e_{\alpha}$ is a holomorphic frame for $L$ over an open set $U_{\alpha}$ then $\left|e_{\alpha}\right|_{h}=e^{-\varphi_{\alpha}}$ where $\varphi_{\alpha} \in \mathscr{C}^{2}\left(U_{\alpha}\right)$ such that $\varphi_{\alpha}=\varphi_{\beta}+\log \left|g_{\alpha \beta}\right|$ and $g_{\alpha \beta}:=e_{\beta} / e_{\alpha} \in \mathcal{O}^{*}\left(U_{\alpha} \cap U_{\beta}\right)$ are the transition functions for $L$. Then one can define global extremal weight $\varphi_{e}$ to be

$$
\varphi_{e}:=\sup \{\psi \text { is a psh weight }: \psi \leq \varphi \text { on } M\} .
$$

It follows that $\varphi_{e}$ defines a psh weight of the Hermitian metric $h_{e}:=e^{-\varphi_{e}}$ on $L$. We denote its curvature current by $d d^{c} \varphi_{e}:=d d^{c}\left(\varphi_{e, \alpha}\right)$ on $U_{\alpha}$. Note that by the compatibility condition we have $\varphi_{e, \alpha}=\varphi_{e, \beta}+\log \left|g_{\alpha \beta}\right|$ and the current $d d^{c} \varphi_{e}$ is a globally well-defined positive closed $(1,1)$ current on $M$. Moreover, by [Ber09a] the equilibrium measure

$$
\mu_{\varphi_{e}}:=\left(d d^{c} \varphi_{e}\right)^{m} / m !
$$

is supported on the compact set

$$
S_{\varphi}:=M_{\varphi}(0) \cap D
$$

where $M_{\varphi}(0):=\left\{x \in M: d d^{c} \varphi(x)>0\right\}$ and $D:=\left\{x \in M: \varphi(x)=\varphi_{e}(x)\right\}$. 
The geometric data given above allow us to define a scalar inner product on the vector space of global holomorphic sections $H^{0}\left(M, L^{\otimes n}\right)$ via

$$
\left\langle s_{1}, s_{2}\right\rangle:=\int_{X}\left\langle s_{1}(x), s_{2}(x)\right\rangle_{h^{\otimes n}} d V
$$

where $d V$ is a fixed volume form on $M$. We also denote the induced norm by $\|\cdot\|_{n}$. Next, we fix an ONB $\left\{S_{j}^{n}\right\}_{j=1}^{d_{n}}$ for $H^{0}\left(M, L^{\otimes n}\right)$ with respect to the inner product (5.2). Then a subgaussian random holomorphic section is of the form

$$
S_{n}:=\sum_{j=1}^{d_{n}} c_{j}^{n} S_{j}^{n}
$$

where $c_{j}^{n}$ are iid (real or complex) subgaussian random variables. This definition induces a $d_{n^{-}}$ fold product probability measure $\operatorname{Prob}_{n}$ on the vector space $H^{0}\left(M, L^{\otimes n}\right)$. We also consider the product probability space $\prod_{n=1}^{\infty}\left(H^{0}\left(M, L^{\otimes n}\right), \operatorname{Prob}_{n}\right)$. The arguments in $\S 3$ carries over to the current geometric setting, in particular almost every sequence of subgaussian random holomorphic sections is quantum ergodic in the sense of [Zel18]:

Theorem 5.1. Let $M$ be a projective complex manifold and $(L, h)$ be an ample Hermitian holomorphic line bundle endowed with a $\mathscr{C}^{2}$ metric $h$. Then for almost every sequence in $\prod_{n=1}^{\infty}\left(H^{0}\left(M, L^{\otimes n}\right)\right.$, Prob $)$ the masses

$$
\frac{1}{d_{n}}\left|s_{n}(z)\right|_{h^{\otimes n}}^{2} d V \rightarrow d \mu_{\varphi_{e}}
$$

in the weak-star sense on $S_{\varphi}$. Moreover, almost surely in $\prod_{n=1}^{\infty}\left(H^{0}\left(M, L^{\otimes n}\right)\right.$, Prob $\left._{n}\right)$ the normalized currents of integration

in the sense of currents.

$$
\frac{1}{n}\left[Z_{s_{n}}\right] \rightarrow d d^{c} \varphi_{e}
$$

\section{REFERENCES}

[Bay16] T. Bayraktar. Equidistribution of zeros of random holomorphic sections. Indiana Univ. Math. J., 65(5):17591793, 2016.

[Bay17a] T. Bayraktar. Asymptotic normality of linear statistics of zeros of random polynomials. Proc. Amer. Math. Soc., 145(7):2917-2929, 2017.

[Bay17b] T. Bayraktar. Zero distribution of random sparse polynomials. Michigan Math. J., 66(2):389-419, 2017.

[Bay19] T. Bayraktar. On global universality for zeros of random polynomials. Hacet. J. Math.Stat., 48(2):384-398, 2019.

[BCHM18] T. Bayraktar, D. Coman, H. Herrmann, and G. Marinescu. A survey on zeros of random holomorphic sections. Dolomites Res. Notes Approx., 11(4):1-19, 2018.

[BCM] T. Bayraktar, D. Coman, and G. Marinescu. Universality results for zeros of random holomorphic sections. Trans. Amer. Math. Soc., (DOI:10.1090/tran/7807).

[Ber09a] R. J. Berman. Bergman kernels and equilibrium measures for line bundles over projective manifolds. Amer. J. Math., 131(5):1485-1524, 2009.

[Ber09b] R. J. Berman. Bergman kernels for weighted polynomials and weighted equilibrium measures of $\mathbb{C}^{n}$. Indiana Univ. Math. J., 58(4):1921-1946, 2009.

[BD18] T. Bloom and D. Dauvergne. Asymptotic zero distribution of random orthogonal polynomials. Ann. Probab., 47(5):3202-3230, 2019.

[BL15] T. Bloom and N. Levenberg. Random Polynomials and Pluripotential-Theoretic Extremal Functions. Potential Anal., 42(2):311-334, 2015.

[Blo01] T. Bloom. On families of polynomials which approximate the pluricomplex Green function. Indiana Univ. Math. J., 50(4):1545-1566, 2001.

[Blo05] T. Bloom. Random polynomials and Green functions. Int. Math. Res. Not., (28):1689-1708, 2005. 
[BLPW15] T. Bloom, N. Levenberg, F. Piazzon, and F. Wielonsky. Bernstein-markov: a survey. Dolomites Research Notes on Approximation, 8(Special_Issue), 2015.

[BS07] T. Bloom and B. Shiffman. Zeros of random polynomials on $\mathbb{C}^{m}$. Math. Res. Lett., 14(3):469-479, 2007.

[BT76] E. Bedford and B. A. Taylor. The Dirichlet problem for a complex Monge-Ampère equation. Invent. Math., 37(1):1-44, 1976.

[BT82] E. Bedford and B. A. Taylor. A new capacity for plurisubharmonic functions. Acta Math., 149(1-2):1-40, 1982.

[BT87] E. Bedford and B. A. Taylor. Fine topology, šilov boundary, and $\left(d d^{c}\right)^{n}$. J. Funct. Anal., 72(2):225-251, 1987.

[Dem09] J.-P. Demailly. Complex analytic and differential geometry. http://www-fourier.ujf-grenoble.fr/ demailly/manuscripts/agbook.pdf, 2009.

[Din09] S. Dinew. Uniqueness in $\mathscr{E}(X, \omega) . J$. Funct. Anal., 256(7):2113-2122, 2009.

[DS06] T.-C. Dinh and N. Sibony. Distribution des valeurs de transformations méromorphes et applications. Comment. Math. Helv., 81(1):221-258, 2006.

[ET50] P. Erdös and P. Turán. On the distribution of roots of polynomials. Ann. of Math. (2), 51:105-119, 1950.

[GZ07] V. Guedj and A. Zeriahi. The weighted Monge-Ampère energy of quasiplurisubharmonic functions. J. Funct. Anal., 250(2):442-482, 2007.

[H9̈4] L. Hörmander. Notions of convexity, volume 127 of Progress in Mathematics. Birkhäuser Boston, Inc., Boston, MA, 1994.

[Ham56] J. M. Hammersley. The zeros of a random polynomial. In Proceedings of the Third Berkeley Symposium on Mathematical Statistics and Probability, 1954-1955, vol. II, pages 89-111, Berkeley and Los Angeles, 1956. University of California Press.

[HN08] C. P. Hughes and A. Nikeghbali. The zeros of random polynomials cluster uniformly near the unit circle. Compos. Math., 144(3):734-746, 2008.

[HW71] D. L. Hanson and F. T. Wright. A bound on tail probabilities for quadratic forms in independent random variables. Ann. Math. Statist., 42:1079-1083, 1971.

[IZ13] I. Ibragimov and D. Zaporozhets. On distribution of zeros of random polynomials in complex plane. In Prokhorov and Contemporary Probability Theory, pages 303-323. Springer, 2013.

[Kac43] M. Kac. On the average number of real roots of a random algebraic equation. Bull. Amer. Math. Soc., 49:314320, 1943.

[Kli91] M. Klimek. Pluripotential theory, volume 6 of London Mathematical Society Monographs. New Series. The Clarendon Press, Oxford University Press, New York, 1991. Oxford Science Publications.

[LO43] J. E. Littlewood and A. C. Offord. On the number of real roots of a random algebraic equation. III. Rec. Math. [Mat. Sbornik] N.S., 12(54):277-286, 1943.

[NV98] S. Nonnenmacher and A. Voros. Chaotic eigenfunctions in phase space. J. Statist. Phys., 92(3-4):431-518, 1998.

[Pri18] I. E. Pritsker. Zero distribution of random polynomials. J. Anal. Math., 134(2):719-745, 2018.

[Rud05] Z. Rudnick. On the asymptotic distribution of zeros of modular forms. Int. Math. Res. Not., (34):2059-2074, 2005.

[RV13] M. Rudelson and R. Vershynin. Hanson-Wright inequality and sub-Gaussian concentration. Electron. Commun. Probab., 18:no. 82, 9, 2013.

[Sic81] J. Siciak. Extremal plurisubharmonic functions in $\mathbf{C}^{n}$. Ann. Polon. Math., 39:175-211, 1981.

[ST97] E. B. Saff and V. Totik. Logarithmic potentials with external fields, volume 316 of Grundlehren der Mathematischen Wissenschaften [Fundamental Principles of Mathematical Sciences]. Springer-Verlag, Berlin, 1997. Appendix B by Thomas Bloom.

[SV95] L. A. Shepp and R. J. Vanderbei. The complex zeros of random polynomials. Trans. Amer. Math. Soc., 347(11):4365-4384, 1995.

[SZ99] B. Shiffman and S. Zelditch. Distribution of zeros of random and quantum chaotic sections of positive line bundles. Comm. Math. Phys., 200(3):661-683, 1999.

[Ver12] R. Vershynin. Introduction to the non-asymptotic analysis of random matrices. In Compressed sensing, pages 210-268. Cambridge Univ. Press, Cambridge, 2012.

[Wal82] P. Walters. An introduction to ergodic theory, volume 79 of Graduate Texts in Mathematics. Springer-Verlag, New York, 1982.

[Zel18] S. Zelditch. Quantum ergodic sequences and equilibrium measures. Constr. Approx., 47(1):89-118, 2018.

FACUlty of ENGINEERING AND NATURAl Sciences, SABANCi University, İstanbul, TuRKey

Email address: tbayraktar@sabanciuniv.edu 